\title{
Loperamide Abuse: A Case Report and Brief Review
}

\author{
Jaclyn Leong $D O^{1}$ \\ Kava Afu MS-32 \\ Ella Starobinska MD ${ }^{1}$ \\ Michael Insel MD \\ ${ }^{1}$ Department of Internal Medicine, ${ }^{2}$ University of Arizona College of Medicine, and \\ ${ }^{3}$ Department of Pulmonary and Critical Care \\ Banner University Medical Center \\ Tucson, AZ USA
}

\section{Case Presentation}

A 29-year-old man with unspecified mood disorder, childhood attention deficit hyperactivity disorder, and 2 prior suicide attempts with zolpidem and methadone presented with altered mental status as a transfer from an outside hospital. The patient was found in his truck outside of a grocery store by a bystander who contacted emergency medical services. At the time, he was noted to have seizure-like activity. He had an empty shopping bag in his possession with 14 empty loperamide (Imodium $\AA$ ) bottles, each were supposed to contain 24 tablets. The patient was taken to the nearest medical facility where he was found to be in status epilepticus. After no response to 4 $\mathrm{mg}$ intravenous (IV) lorazepam, he was then intubated for airway protection. Propofol infusion was started and a loading dose of levetiracetam $1600 \mathrm{mg}$ IV was administered. Despite medical management, he continued to show evidence of seizure-like activity. Due to the lack of a neurology service at the hospital, the patient was transferred to our academic medical center.

On arrival, patient was intubated, sedated and hemodynamically stable. Sedation was paused to allow a thorough neurologic examination, at which time, he became agitated and was not redirectable. However, he did not exhibit seizure activity. Toxicology service was consulted for suspected loperamide overdose.

Laboratory workup revealed white blood cell count $16,600 \mu \mathrm{L}$ with neutrophilic predominance (4000-11000), glucose $205(70-106 \mathrm{mg} / \mathrm{dL})$, lactic acid of 10 (0.5- 1.7 $\mathrm{mmol} / \mathrm{L})$, creatinine kinase 164 (35- $232 \mathrm{IU} / \mathrm{L})$, creatinine 1.39 (0.60- $1.50 \mathrm{mg} / \mathrm{dL})$, EGFR 79 (Normal Low >=60). Tylenol and salicylate levels were undetectable. Urine drug screen was negative. Computer tomography (CT) of the head showed no acute intracranial process. CT abdomen/pelvis showed bibasilar airspace consolidations concerning for aspiration pneumonia. Echo revealed left ventricular ejection fraction of $38 \%$ with a large-sized apical, septal, anteroseptal, anterior, inferior, posterior, and lateral wall motion abnormality with hypokinesis to akinesis of the segments. 
Electrocardiograms (ECG) initially showed QTc and QRS prolongation of $571 \mathrm{~ms}$ and $160 \mathrm{~ms}$, respectively. During the initial 24-hours at our hospital, serial EKGs were performed to monitor QTc. Additionally, patient was treated empirically for aspiration pneumonia with ampicillin/sulbactam.

On day two of his hospitalization, his ECG revealed sinus rhythm, with first-degree AV block and QT prolongation greater than $700 \mathrm{~ms}$. Subsequently, torsades de pointes developed, progressing into ventricular tachycardia storm. Several ampules of bicarbonate were administered, and the patient was cardioverted. Dobutamine and magnesium infusions were started and a temporary transvenous pacer was placed with overdrive pacing initiated at $110 \mathrm{bpm}$. Over the next 24 hours, magnesium and dobutamine were discontinued. The transvenous pacer was removed on hospital day 4 after the patient demonstrated a native rhythm with normal QRS and QT intervals. Levetiracetam was also discontinued, per neurology recommendations. The patient was safely extubated on hospital day 5 . He was subsequently evaluated by the psychiatry service who scheduled close follow up after discharge.

\section{Discussion}

Loperamide is a nonprescription drug used most commonly to control acute, nonspecific diarrhea, as well as chronic diarrhea associated with inflammatory bowel disease. It acts on mu-opioid receptors in the myenteric plexus to reduce peristaltic activity, thus lengthening bowel transit time and lowering volume and frequency of bowel movements (1). In contrast to other opioid receptor agonists, loperamide has poor absorption from the gastrointestinal tract and limited ability to cross the blood-brain barrier $(1,2)$. Consequently, the drug was deemed low risk for physical dependence and abuse and since 1982 has been sold over-the-counter (OTC) (3).

Despite this labeling, there is an increasing number of reports documenting cases of loperamide misuse and abuse (3). It has only recently been discovered that loperamide is being ingested at supratherapeutic doses (>16 mg/day) for its euphoric effects or for the relief of opioid withdrawal symptoms $(3,4)$. In 2013 , online reports of recreational loperamide use at doses of $70-100 \mathrm{mg}$ began circulating (3). In the following three years, a $71 \%$ increase of loperamide-associated presentations to drug and poison control agencies was reported $(3,6)$.

As a result, the cardiotoxic effects of this drug have come to light, particularly QTprolongation and QRS- widening (3). At supratherapeutic plasma concentrations, loperamide causes a blockade of the human ether-a-go-go-related gene ( $h E R G$ ) cardiac potassium channel with high affinity, delaying repolarization of the cardiac myocytes and affecting QT-interval and QRS complex (5). Life-threatening dysrhythmias ensue, accounting for the increasing rate of deaths associated with loperamide overdose and toxicity (3). Reports of loperamide-associated cardiac toxicity have increased greatly in number over the past few years and include: at least 21 individual published case reports of loperamide cardiotoxicity, including one published 
in SWJPCC (3,7); 48 cases of serious loperamide-associated cardiac events, identified in the FDA Adverse Event Reporting System database; and 22 cases of patients found dead with elevated plasma concentrations of loperamide (3).

Loperamide is just one of an increasing number of OTC drugs with potential abuse because of the stimulant or sedative effects. Other OTC drugs commonly known for abuse are dextromethorphan, pseudoephedrine, phenylephrine, diphenhydramine and oxybutynin especially among teenagers, however, loperamide is a less commonly known drug for its opioid abuse. It is important for physicians to be aware of this increasing risk of abuse and significant life-threatening cardiotoxic effects.

\section{References}

1. Killinger JM, Weintraub HS, Fuller BL. Human pharmacokinetics and comparative bioavailability of loperamide hydrochloride. J Clin Pharmacol. 1979;19:211-8. [CrossRef] [PubMed]

2. Regnard C, Twycross R, Mihalyo M, et al. Loperamide. J Pain Symptom Manage. 2011;42:319-23.[CrossRef] [PubMed]

3. Wu PE, Juurlink DN. Clinical review: Loperamide toxicity. Annals of Emergency Med. 2017;70:245-52. [CrossRef] [PubMed]

4. Daniulaityte R, Carlson R, Falck R, et al. "I just wanted to tell you that loperamide will work": a web-based study of extra-medical use of loperamide. Drug Alcohol Depend. 2013;130:241-4. [CrossRef] [PubMed]

5. Salama A, Levin Y, Jha P, et al. Ventricular fibrillation due to overdose of loperamide, the "poor man's methadone." J Community Hosp Intern Med Perspect. 2017;7(4):222-6. [CrossRef] [PubMed]

6. Stanciu CN, Gnanasegaram SA. Loperamide, the "Poor Man's Methadone": Brief review. Journal of Psychoactive Drugs. 2017;49:18-21. [CrossRef] [PubMed]

7. Watkins SA, Smelski G, French RNE, Insel M, Campion J. January 2019 critical care case of the month: A 32-year-old woman with cardiac arrest. Southwest J Pulm Crit Care. 2019;18(1):1-7. [CrossRef] 